\title{
\begin{tabular}{l|l} 
Mibraries & DSpace@MIT
\end{tabular}
}

\author{
MIT Open Access Articles
}

\section{The interaction of a walking droplet and a submerged pillar: From scattering to the logarithmic spiral}

The MIT Faculty has made this article openly available. Please share how this access benefits you. Your story matters.

Citation: Harris, Daniel M. et al. "The interaction of a walking droplet and a submerged pillar: From scattering to the logarithmic spiral." Chaos 28, 9 (September 2018): 096105 (c) 2018 Author(s)

As Published: http://dx.doi.org/10.1063/1.5031022

Publisher: AIP Publishing

Persistent URL: https://hdl.handle.net/1721.1/123530

Version: Final published version: final published article, as it appeared in a journal, conference proceedings, or other formally published context

Terms of Use: Article is made available in accordance with the publisher's policy and may be subject to US copyright law. Please refer to the publisher's site for terms of use. 
The interaction of a walking droplet and a submerged pillar: From scattering to the logarithmic spiral

Daniel M. Harris, P.-T. Brun, Adam Damiano, Luiz M. Faria, and John W. M. Bush

Citation: Chaos 28, 096105 (2018); doi: 10.1063/1.5031022

View online: https://doi.org/10.1063/1.5031022

View Table of Contents: http://aip.scitation.org/toc/cha/28/9

Published by the American Institute of Physics

Chaos

An Interdisciplinary Journal of Nonlinear Science

Fast Track Your Research. Submit Today! 


\title{
The interaction of a walking droplet and a submerged pillar: From scattering to the logarithmic spiral
}

\author{
Daniel M. Harris, ${ }^{1,2}$ P.-T. Brun, ${ }^{1,3}$ Adam Damiano, ${ }^{1}$ Luiz M. Faria, ${ }^{1}$ and John W. M. Bush ${ }^{1}$ \\ ${ }^{1}$ Department of Mathematics, Massachusetts Institute of Technology, Cambridge, Massachusetts 02139, USA \\ ${ }^{2}$ School of Engineering, Brown University, Providence, Rhode Island 02912, USA \\ ${ }^{3}$ Department of Chemical and Biological Engineering, Princeton University, Princeton, New Jersey 08540, USA
}

(Received 27 March 2018; accepted 22 June 2018; published online 14 September 2018)

\begin{abstract}
Millimetric droplets may walk across the surface of a vibrating fluid bath, propelled forward by their own guiding or "pilot" wave field. We here consider the interaction of such walking droplets with a submerged circular pillar. While simple scattering events are the norm, as the waves become more pronounced, the drop departs the pillar along a path corresponding to a logarithmic spiral. The system behavior is explored both experimentally and theoretically, using a reduced numerical model in which the pillar is simply treated as a region of decreased wave speed. A trajectory equation valid in the limit of weak droplet acceleration is used to infer an effective force due to the presence of the pillar, which is found to be a lift force proportional to the product of the drop's walking speed and its instantaneous angular speed around the post. This system presents a macroscopic example of pilot-wave-mediated forces giving rise to apparent action at a distance. Published by AIP Publishing. https://doi.org/10.1063/1.5031022
\end{abstract}

\begin{abstract}
A small droplet can bounce indefinitely on a vibrated liquid bath and move horizontally through interactions with its own wave field. In the absence of boundaries, these walking droplets walk in a straight line at a constant speed. In the present study, we demonstrate that the presence of a submerged circular pillar can dramatically alter the trajectory through its influence on the droplet's guiding wave field. Specifically, the wave-mediated interaction between the submerged pillar and the droplet can lead to simple scattering events or to the droplet departing the pillar along a logarithmic spiral.
\end{abstract}

\section{INTRODUCTION}

The logarithmic spiral is a self-similar curve arising in myriad settings in nature and classical mechanics. It has fascinated generations of scientists, including Jacob Bernoulli, who referred to the form as Spira Mirabelis and requested that it be inscribed on his gravestone. Logarithmic spirals arise naturally in the form of nautilus shells, the seed patterns in the heart of the sunflower and the arms of spiral galaxies. They may also arise in pursuit problems, for example, when a hawk pursues its prey while maintaining a fixed angle to its flight path. ${ }^{1}$ When a spinning ball in flight maintains a constant angular speed while decelerating due to form drag, the resulting form is a logarithmic spiral. ${ }^{2}$ Dirac's classical theory of radiating electrons predicts that an electron will follow a logarithmic spiral in the presence of a uniform magnetic field. ${ }^{3}$ We here report an unexpected new example of the emergence of a logarithmic spiral in a hydrodynamic pilot-wave system.

Couder et al. ${ }^{4}$ discovered that a millimetric drop placed on the surface of a vibrating bath may interact with its own wave field in such a way as to walk steadily across the bath. ${ }^{5}$ The resulting "walkers" are spatially extended objects comprising both droplet and accompanying guiding or "pilot" wave. By virtue of their spatial delocalization, the walkers exhibit several dynamical features previously thought to be peculiar to the microscopic realm, including tunneling, 6,7 quantized orbits ${ }^{8,9}$ and orbital level splitting in a rotating frame,${ }^{10}$ doubly quantized orbits in a harmonic potential, ${ }^{11,12}$ and wave-like statistics in confined geometries. ${ }^{13-15}$ The system has recently been reviewed and its relation to the modern extensions of de Broglie's double-solution pilot-wave mechanics explored. ${ }^{16,17}$ We here demonstrate that pilotwave-mediated local forces can give rise to apparent action at a distance when the walking droplets interact with submerged circular barriers.

\section{EXPERIMENTAL SETUP}

In our experiments, a bath of silicon oil of viscosity $v=$ $20 \mathrm{cSt}$, density $\rho=950 \mathrm{~kg} \mathrm{~m}^{-3}$, and surface tension $\sigma=20$ $\mathrm{mN} / \mathrm{m}$ was vibrated sinusoidally at frequency $f=\omega_{0} / 2 \pi=$ $80 \mathrm{~Hz}$ with peak acceleration $\gamma$ [see Fig. 1(a) and Ref. 18 for details]. The bath is of diameter $16 \mathrm{~cm}$ and is surrounded by a shallow border of width $9 \mathrm{~mm}$ and depth $1 \mathrm{~mm}$ that serves to damp waves locally. The bath acceleration $\gamma$ is always below the Faraday threshold $\gamma_{F} \approx 4.2 \mathrm{~g}$, at which the interface becomes unstable to a field of Faraday waves with wavelength $\lambda_{F}$ prescribed by the standard water-wave dispersion relation. ${ }^{19}$ Consequently, the bath would remain quiescent in the absence of the droplet. Droplets of radius $R$ are generated using piezoelectric actuation. ${ }^{20}$ The corresponding vibration number $\omega_{0} / \sqrt{\sigma /\left(\rho R^{3}\right)}$ ranges from 0.67 to 0.81 in our study and prescribes the relative magnitudes of the driving acceleration and the drop's natural frequency. ${ }^{21,22}$ The oil layer has depth $h_{0}$ everywhere except above the pillar, where the depth is $h_{1}$. The pillar diameter, $d$, is varied from $1 \mathrm{~mm}$ to $15 \mathrm{~mm}$. A submerged droplet "launcher" guides the droplet toward the pillar. ${ }^{23}$ Previous studies have indicated the importance of working in a isolated environment to avoid the influence of 


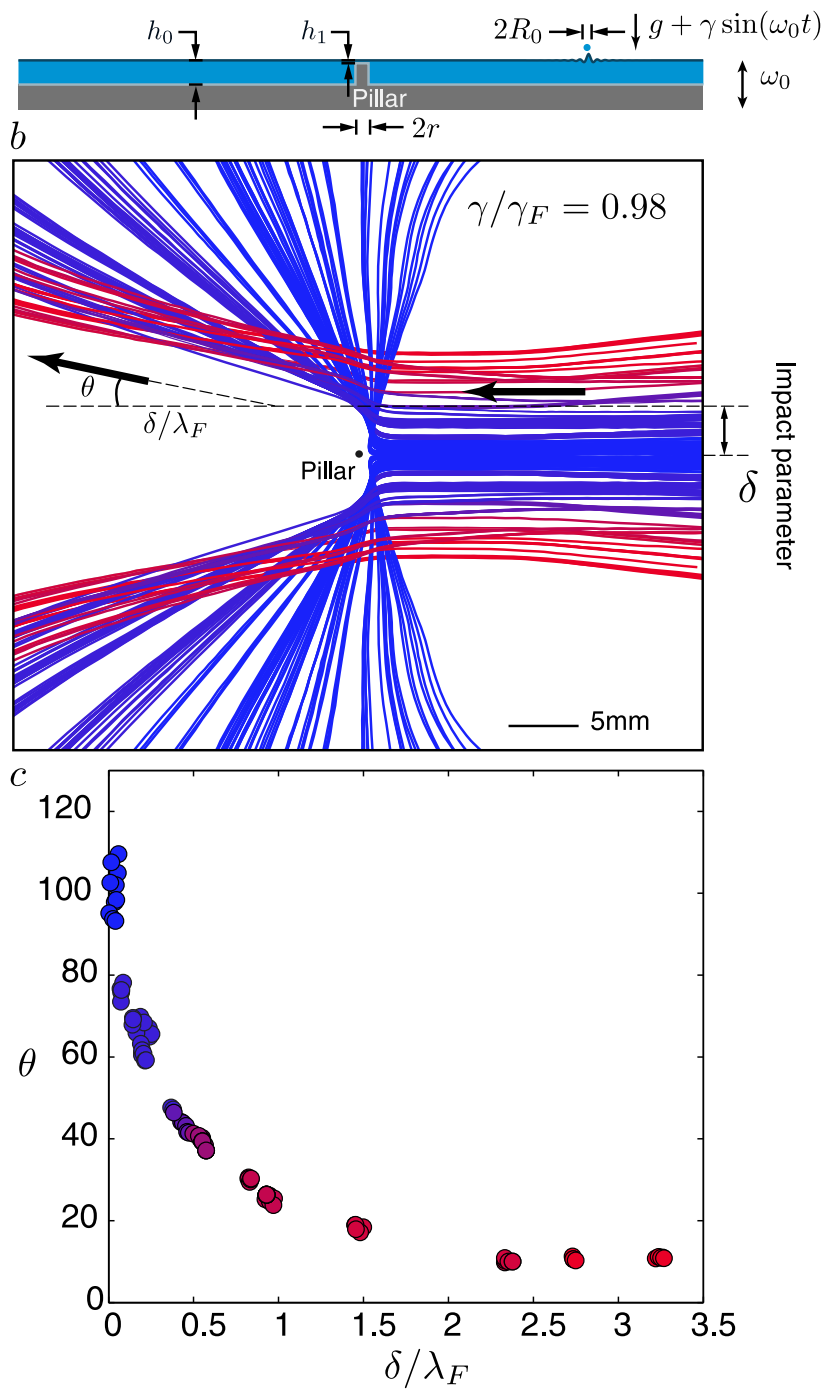

FIG. 1. (a) A fluid bath vibrates vertically with acceleration $\gamma \sin \omega_{0} t$. The depth of the bath varies from $h_{0}$ in the deep region to $h_{1}$ over the pillar. (b) Trajectories of a walking droplet interacting with a submerged pillar of diameter $d=1 \mathrm{~mm}$ at a fixed vibrational forcing $\gamma / \gamma_{F}=0.98$. For these data, $R=0.38 \mathrm{~mm}, h_{0}=10 \mathrm{~mm}$, and $h_{1}=0.3 \mathrm{~mm}$. (c) Dependence of scattering angle $\theta$ on impact parameter $\delta$.

ambient air currents, ${ }^{23}$ so we use a sealed transparent lid on the vibrating container.

\section{SCATTERING}

For the smallest pillar examined, $d=1 \mathrm{~mm}$, pure scattering trajectories were observed [Fig. 1(b)]. The scattering angle $\theta$, defined as the deviation from the incoming trajectory, decreases monotonically with increasing impact parameter $\delta$ defined as the perpendicular distance between the initial droplet path and the pillar center [Fig. 1(c)]. For head-on collisions $\delta \simeq 0$, the drop is deflected such that $\theta>90^{\circ}$. The scattering angle then rapidly decreases with increasing $\delta$. For the largest impact parameters considered, $\delta \simeq 3.5 \lambda_{F}$, the drop still interacts with the pillar, and $\theta \simeq 10^{\circ}$, underscoring the relatively long-range dynamical influence of the pillar as communicated by the droplet's pilot wave field. We note that the observed behavior might be characterized in terms of elastic scattering, insofar as the incoming and outgoing drop speeds are identical. While the scattering behavior observed for the smallest pillar suggests that the effect of the pillar is repulsive, we shall proceed by demonstrating that, for larger pillars, an initial repulsion may be followed by an attractive tethering phase.

\section{THE LOGARITHMIC SPIRAL}

Figure 2(a) illustrates the trajectories arising from scattering from a pillar with diameter $d=5 \mathrm{~mm}$ for a fixed impact parameter and different values of $\gamma / \gamma_{F}$ ranging from $97 \%$ to $99 \%$. In all cases, the drop is initially repelled by the pillar, from a point of closest approach $r_{\min }$ that depends weakly on $\gamma / \gamma_{F}$. Thereafter, the drops follow a logarithmic spiral with constant radial velocity $v_{r}$, azimuthal velocity $v_{\theta}$, and speed $|\mathbf{v}|=\sqrt{v_{r}^{2}+v_{\theta}^{2}}$. The parametric equation for the trajectory is

$$
r(\theta)=r_{\min } e^{\frac{v_{r}}{v_{\theta}}\left(\theta-\theta_{0}\right)},
$$
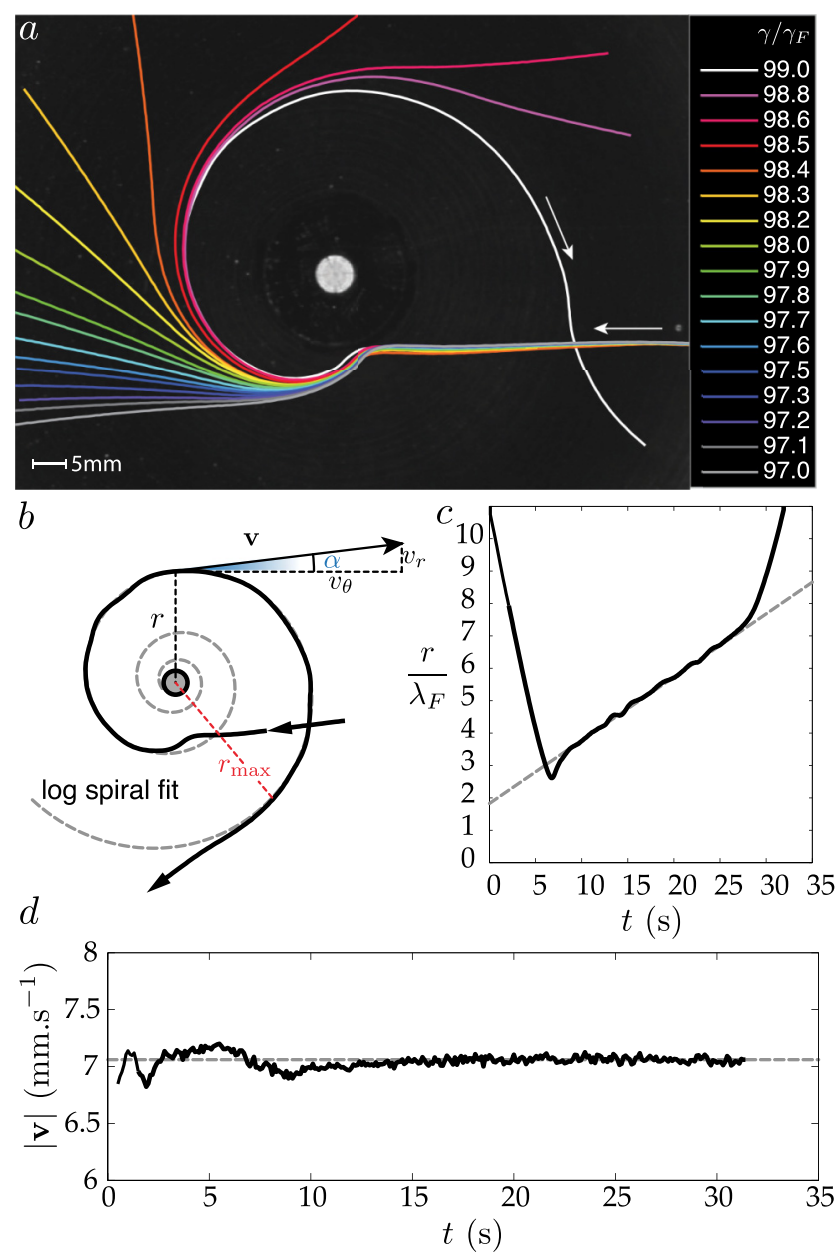

FIG. 2. (a) Observed variation of droplet trajectories as the dimensionless vibrational acceleration, $\gamma / \gamma_{F}$, increases from $97 \%$ to $99 \%$. Pillar diameter is $d=5 \mathrm{~mm}$. (b) Individual trajectories are fitted to a logarithmic spiral with pitch angle $\alpha=\arctan \left(v_{r} / v_{\theta}\right)$. The time evolution of (c) the drop's distance from the pillar center and (d) the droplet speed. When locked onto the spiral trajectory, the droplet moves at a nearly constant speed corresponding to its free walking speed $u_{0}$. Drop diameter, $R=0.34 \mathrm{~mm}, h_{0}=5.24 \mathrm{~mm}, h_{1}=$ $0.46 \mathrm{~mm}$. In (b)-(d), $\gamma / \gamma_{F}=0.995$. 


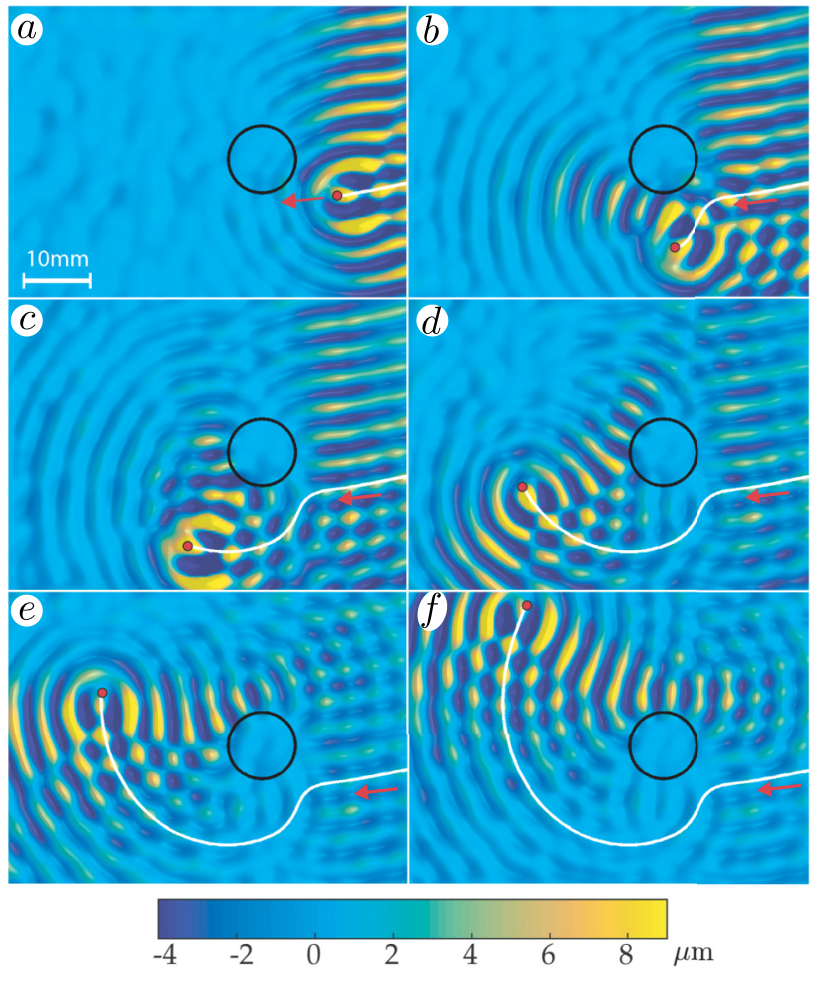

FIG. 3. Surface topography measurements ${ }^{26}$ of a bouncing droplet with radius and driving acceleration $\gamma / \gamma_{F}=0.995$ interacting with a pillar with diameter $d=10 \mathrm{~mm}$. The time interval between consecutive images is $1.625 \mathrm{~s}$. The white line indicates the trajectory traced out by the walking droplet, which changes from rectilinear motion to curvilinear motion along a logarithmic spiral. Images correspond to the bath at its maximum vertical displacement. $R=0.38 \mathrm{~mm}, h_{0}=10 \mathrm{~mm}, h_{1}=0.3 \mathrm{~mm}$. Reprinted by permission from RightsLink Permissions Springer Customer Service Centre GmbH: Springer Nature, Damiano et al., Exp. Fluids 57(10), 163 (2016). Copyright $2016 .^{26}$

where $r\left(\theta_{0}\right)=r_{\min }$ denotes the radius at which the drop locks onto the spiral. The ratio of the radial and azimuthal speeds prescribes the spiral's pitch angle $\alpha=\arctan \left(v_{r} / v_{\theta}\right)$ [see Fig. 2(b)]. The tighter the spiral, the smaller $\alpha$. Beyond a critical escape radius, or tethering length $r_{\max }$, the pillar no longer influences the droplet, which resumes its rectilinear free walking state. In Fig. 2(b), we compare the trajectory observed for $\gamma / \gamma_{F}=0.995$ to a logarithmic spiral of the form (1) with pitch angle $\alpha \simeq 7.8^{\circ}$ as inferred from speed measurements $\left(v_{r}=0.96 \mathrm{~mm} \mathrm{~s}^{-1}\right.$ and $\left.v_{\theta}=6.9 \mathrm{~mm} \mathrm{~s}^{-1}\right)$. We note that the instantaneous angular speed of the drop around the pillar, $\Omega=v_{\theta} / r$, decreases as $1 / r$ given the constancy of $v_{\theta}$.

The pillar affects the droplet's trajectory exclusively through its influence on the pilot wave field. It is thus illuminating to measure the evolution of the wave field during the droplet-pillar interaction using the surface synthetic Schlieren technique. ${ }^{25,26}$ In Fig. 3, we report the surface topography of a drop interacting with a pillar of diameter $d=10 \mathrm{~mm}$. Note that the peak amplitude of the waves, $10 \mu \mathrm{m}$, is reduced to less than $1 \mu \mathrm{m}$ above the pillar. The abrupt change of the wave properties above the pillar is a key feature of this problem to be captured in our numerical simulations. Note also the band of relatively intense wave activity on the "tethering line" that may be drawn between the droplet and the pillar.
We proceed by characterizing how the features of such spirals depend on the system parameters. In Fig. 4(a) we show the dependence of the pitch angle $\alpha$ on $\gamma / \gamma_{F}$ for the experimental trajectories displayed in Fig. 2(a). While changing $\gamma$ has little effect on the drop speed $|\mathbf{v}|$, it does alter the relative magnitudes of $v_{r}$ and $v_{\theta}$. The pitch angle is thus found to decrease with increasing $\gamma / \gamma_{F}$, so the spiral becomes tighter as the waves become more pronounced. We note that the case of a circular trajectory, which would correspond to pillar-induced trapping of the droplet, was not achieved in our experiments.

In Fig. 4(a), we also report the dependence of the tethering length $r_{\max }$ on $\gamma / \gamma_{F}$. We note that the abrupt increase in $r_{\max }$ above $\gamma / \gamma_{F}=0.982$ is likely related to the rapid increase in the spatial extent of the pilot-wave field of the walker as the Faraday threshold is approached. ${ }^{24}$ In Fig. 4(b), we see the effect of varying the impact parameter $\delta$ while holding all other parameters constant. Within the range bounded by the dashed lines in Fig. 4(b), we see that all trajectories obtained experimentally may be collapsed onto a single spiral by simple rotation about the center of the pillar [Fig. 4(c)]. The pitch angle $\alpha$ is thus independent of $\delta$ for a pillar of a given size. However, the pitch angle does depend on the diameter $d$ of the pillar. In Fig. 4(d), we show three trajectories observed experimentally with different $d$. The smaller the pillar, the smaller $\alpha$. For $d=3 \mathrm{~mm}$, the droplet executes a complete revolution around the pillar as it drifts away from it, wobbling around the spiral. Once again, the trapping of droplets onto a circular orbit was not observed for any pillar size or driving acceleration. Since further decreasing $d$ ultimately leads to simple scattering events, one can infer that there is a pillar size at which the pitch angle will obtain a minimum value, allowing for the longest possible spiral.

In order to avoid the influence of the bath's outer boundaries, as may become significant for the largest spirals observed in our laboratory study, we employ the numerical model of Faria, ${ }^{27}$ which is able to reliably treat walkerboundary interactions. The model was built from the theoretical model of walking droplets developed by Moláček and Bush $^{21,28}$ and from the wave-field modeling of Milewski et $a .^{24}$ and has been benchmarked against experiment for walker-boundary interactions in a number of settings. ${ }^{23,27,29}$ Our simulations indicate that the pitch angle and tethering length $r_{\max }$ are only weakly dependent on pillar depth $h_{1}$ over the range considered [Figs. 4(e) and 4(f)]: all trajectories lock onto the same spiral, with their time on the spiral increasing as $h_{1}$ decreases. Our simulations also show that the escape radius $r_{\max }$ decreases with $h_{1}$ [Fig. 4(f)]. We note that $r_{\max }$ is independent of the impact parameter $\delta$ provided the drop locks onto a spiral. Our numerical simulations also demonstrated that the spiral induced by a square pillar is similar to that of a circular pillar with the same diameter, suggesting that the spiral and its features are not strongly sensitive to the pillar shape.

We now seek to infer the pillar-induced force. Molácek and $\mathrm{Bush}^{21,28}$ developed a theoretical model for the motion of a drop on a vibrating bath that rationalizes the reported bouncing and walking behaviors. ${ }^{22,30}$ By averaging the forces on the walking droplet over the bouncing period, they developed a trajectory equation describing the drop's horizontal motion 

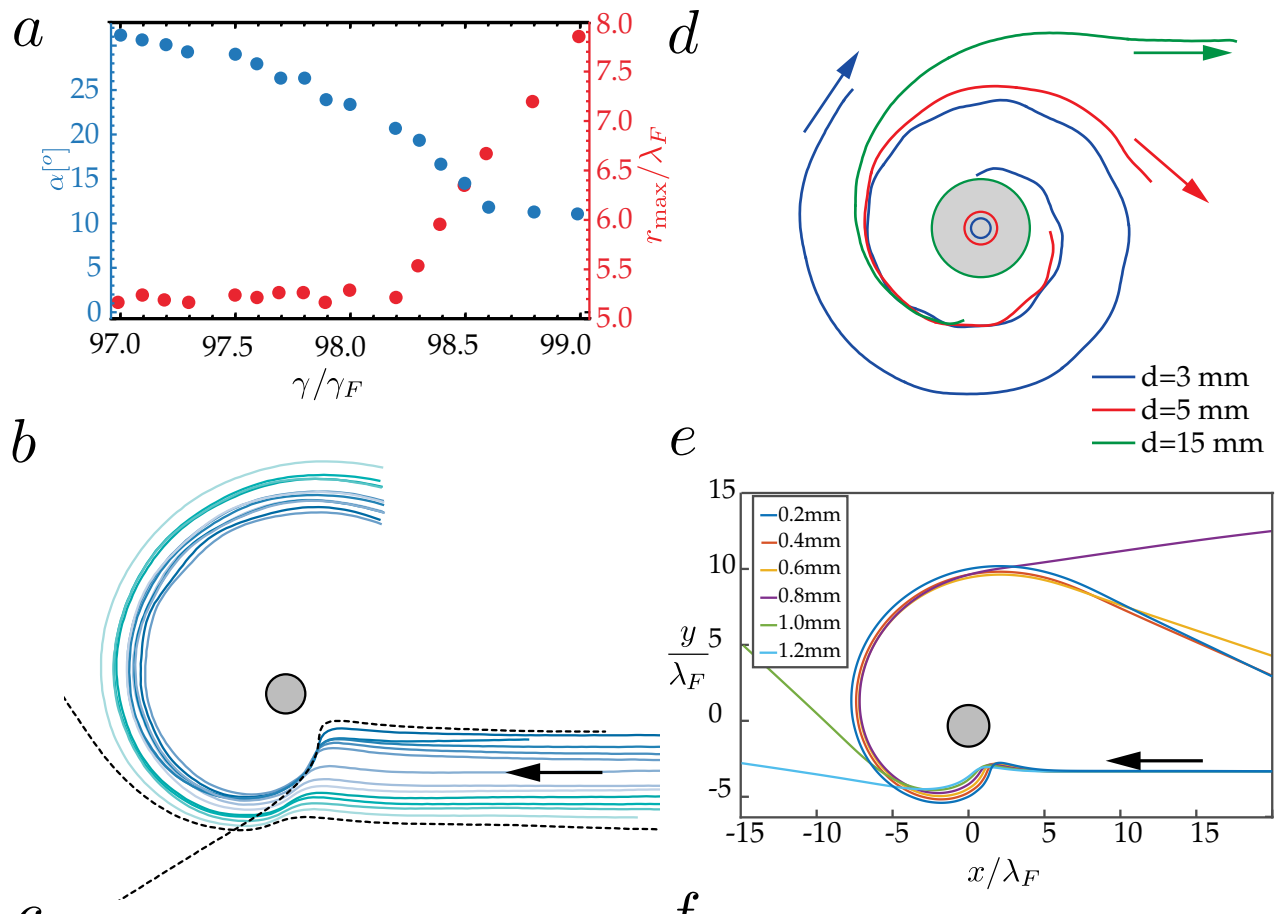

C
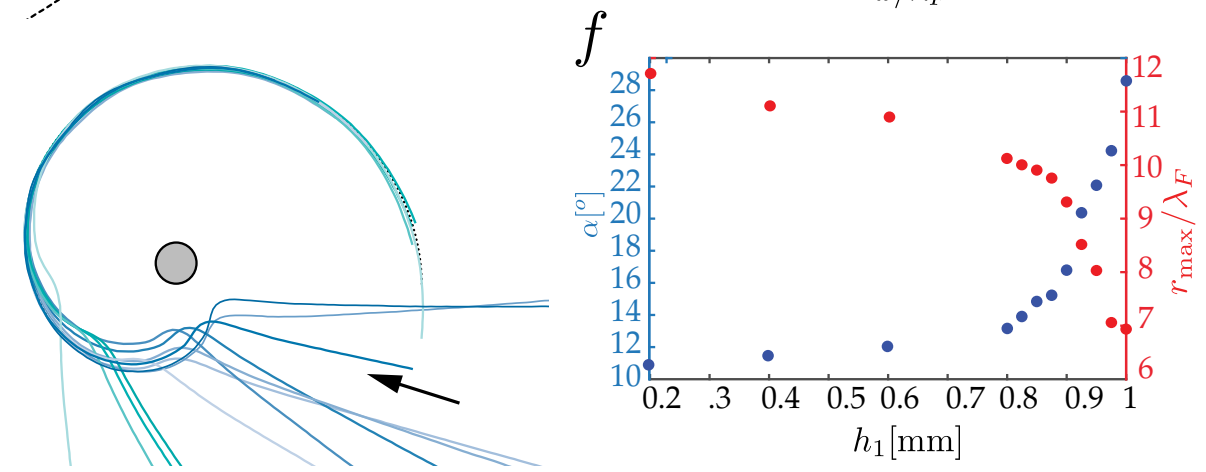

FIG. 4. (a) Observed dependence of pitch angle $\alpha$ and maximum tethering distance $r_{\max }$ on driving acceleration, $\gamma / \gamma_{F}$, at fixed pillar depth $h_{1}=0.3 \mathrm{~mm}$ for a pillar of diameter $d=5 \mathrm{~mm}$. (b) Trajectories within the tethering range, obtained by varying the impact parameter $\delta$, all collapse onto a single spiral (c) upon rotation. Here, $\gamma / \gamma_{F}=0.99$ and $d=5 \mathrm{~mm}$. (d) Spirals obtained experimentally with three different pillar diameters $(d=3,5,15 \mathrm{~mm})$ at the same $\gamma / \gamma_{F}=0.99$ indicate that the pitch angle decreases with increasing $d$. (e) Computed trajectories for a pillar of diameter $d=10 \mathrm{~mm}$, obtained with different pillar depths $h_{1}$. (f) Computed dependence of pitch angle $\alpha$ and maximum tethering distance $r_{\max }$ on pillar depth $h_{1}$. In all simulations, $R=0.38 \mathrm{~mm}, h_{0}=6 \mathrm{~mm}, \gamma / \gamma_{F}=0.99$.

that was recast in an integro-differential form by Oza et al. ${ }^{31}$ Bush et al. ${ }^{17}$ examined the resulting "stroboscopic" model in the limit of weak droplet acceleration, allowing them to recast the trajectory equation in a temporally local form that is valid in the absence of submerged topography. Specifically, asymptotic expansion of the wave force in the weak-acceleration limit demonstrates that the drop motion may be described in terms of the mechanics of a particle with a speed-dependent mass $m \gamma_{B}(v)$ and a nonlinear drag force $D_{w}(v)$ that drives it toward its free walking speed $u_{0} .{ }^{32} \mathrm{We}$ describe the overall effect of the pillar on the drop as an instantaneous force $\mathbf{F}_{p}$ and so write

$$
\frac{d\left(m \gamma_{B} \mathbf{v}\right)}{d t}+D_{w}(v) \mathbf{v}=\mathbf{F}_{p},
$$

where the dependence of the speed-dependent "boost factor" $\gamma_{B}(v)>1$ and the nonlinear drag coefficient $D_{w}$ on the system parameters may be found in Ref. 17.

When the drop walks at its free speed, $v=u_{0}$, the drag coefficient vanishes $D_{w}\left(u_{0}\right)=0$. Consequently, since $|\mathbf{v}|=$ $u_{0}$ along the spiral, we may describe the walker in terms of the inviscid dynamics of a particle whose mass depends on its speed. Specifically,

$$
m \gamma_{B} \frac{d \mathbf{v}}{d t}=\mathbf{F}_{p} .
$$

Inserting trajectory equations consistent with the parametric Eq. (1) and the constancy of $v_{r}$ and $v_{\theta}$, specifically $r(t)=$ $v_{r} t+r_{\min }$ and $\theta(t)=v_{\theta} / v_{r} * \ln \left(v_{r} t+r_{\min }\right)+\theta_{0}$, into Eq. (3) reveals a remarkably simple form for the pillar-induced force:

$$
\mathbf{F}_{p}=m \gamma_{B} \boldsymbol{\Omega} \times \mathbf{v},
$$

where $\boldsymbol{\Omega}=\left(v_{\theta} / r\right) \hat{\mathbf{k}}$ is the instantaneous angular velocity of the drop around the pillar and $\hat{\mathbf{k}}$ is the upward unit vector normal to the undisturbed free surface. We note that, despite the circular symmetry of the system geometry, the pillar-induced force $\mathbf{F}_{p}$ is not radial, but rather orthogonal to the drop trajectory at all times. The lift force so inferred is thus equivalent in form to a Coriolis force acting on the droplet with boosted mass $m \gamma_{B}$. 


\section{CONCLUSION}

We have characterized the impact of a walking droplet on a submerged pillar. While the effect of relatively small pillars is to repel the oncoming drop, leading to a simple scattering event, an unexpected effect arises for larger pillars. Specifically, the droplet locks onto a logarithmic spiral whose form is largely independent over a range of impact parameters, but whose pitch angle decreases with $\gamma / \gamma_{F}$ and pillar size. The boost equation ${ }^{17}$ has allowed us to infer that, through its influence on the pilot wave field, the pillar induces a lift force $\mathbf{F}_{p}=$ $m \gamma_{B} \boldsymbol{\Omega} \times \mathbf{v}$, where $\boldsymbol{\Omega}$ is the instantaneous angular velocity of the pillar around the post. The analogous form of the Coriolis force acting on a mass in a rotating frame and the Lorentz force acting on a charge in a uniform magnetic field ${ }^{8}$ invites the identification of an electromagnetic analog for our system. Dirac's classical theory of radiating electrons predicts that an electron will spiral inwards along a logarithmic spiral in the presence of a uniform magnetic field. ${ }^{3}$ The outward spirals reported here would presumably arise for the case of electron motion in a uniform magnetic field if the electrons were absorbing energy from their environment (and so maintaining a uniform speed) rather than radiating energy into it.

The pilot-wave dynamics of the walking drops are local in that the instantaneous force acting on the drop is determined by the local wave form at the point of impact. However, the dynamics might also be interpreted as non-local in both space and time in the sense that the wave form at the point of impact depends on both the walker's history and its environment. ${ }^{30}$ The logarithmic spiral discovered here provides a new macroscopic example of how a local pilot-wave dynamics can give rise to the inference of a nonlocal force, or how pilot-wavemediated local forces can give rise to apparent action at a distance.

\section{ACKNOWLEDGMENTS}

We thank the NSF for financial support (Grant Nos. CMMI-1727565, CMMI-1333242, and DMS-1614043). We thank Alexis Page for early contributions to the experiments.

${ }^{1}$ V. A. Tucker, A. E. Tucker, K. Akers, and J. H. Enderson, "Curved fligh paths and sideways vision in peregrine falcons (Falco peregrinus)," J. Exp. Biol. 203(24), 3755-3763 (2000), available at http://jeb.biologists. org/content/203/24/3755.short.

${ }^{2}$ G. Dupeux, A. Le Goff, D. Quéré, and C. Clanet, "The spinning ball spiral," New J. Phys. 12(9), 093004 (2010).

${ }^{3} \mathrm{C}$. Jayaratnam Eliezer, Radiating electron in a magnetic field, in Mathematical Proceedings of the Cambridge Philosophical Society (Cambridge University Press, 1946), Vol. 42, pp. 40-44.

${ }^{4}$ Y. Couder, S. Protière, E. Fort, and A. Boudaoud, "Walking and orbiting droplets," Nature 437, 208 (2005).

${ }^{5} \mathrm{~S}$. Protière, A. Boudaoud, and Y. Couder, "Particle-wave association on a fluid interface," J. Fluid Mech. 554, 85-108 (2006).

${ }^{6}$ A. Eddi, E. Fort, F. Moisy, and Y. Couder, "Unpredictable tunneling of a classical wave-particle association," Phys. Rev. Lett. 102, 240401 (2009).
${ }^{7}$ A. Nachbin, P. A. Milewski, and J. W. M. Bush, "Tunneling with a hydrodynamic pilot-wave model," Phys. Rev. Fluids 2(3), 034801 (2017). ${ }^{8}$ E. Fort, A. Eddi, A. Boudaoud, J. Moukhtar, and Y. Couder, "Path-memory induced quantization of classical orbits," Proc. Natl. Acad. Sci. 107(41), 17515-17520 (2010).

${ }^{9}$ D. M. Harris and J. W. M. Bush, "Droplets walking in a rotating frame: From quantized orbits to multimodal statistics," J. Fluid Mech. 739, 444-464 (2014).

${ }^{10}$ A. Eddi, J. Moukhtar, S. Perrard, E. Fort, and Y. Couder, "Level splitting at macroscopic scale," Phys. Rev. Lett. 108(26), 264503 (2012).

${ }^{11}$ S. Perrard, M. Labousse, M. Miskin, E. Fort, and Y. Couder, "Selforganization into quantized eigenstates of a classical wave-driven particle," Nat. Commun. 5 (2014).

${ }^{12}$ M. Labousse, A. U. Oza, S. Perrard, and J. W. M. Bush, "Pilot-wave dynamics in a harmonic potential: Quantization and stability of circular orbits," Phys. Rev. E 93(3), 033122 (2016).

${ }^{13}$ D. M. Harris, J. Moukhtar, E. Fort, Y. Couder, and J. W. M. Bush, "Wavelike statistics from pilot-wave dynamics in a circular corral," Phys. Rev. E 88, 011001 (2013).

${ }^{14} \mathrm{~T}$. Gilet, "Quantumlike statistics of deterministic wave-particle interactions in a circular cavity," Phys. Rev. E 93(4), 042202 (2016).

${ }^{15}$ P. J. Sáenz, T. Cristea-Platon, and J. W. M. Bush, "Statistical projection effects in a hydrodynamic pilot-wave system," Nat. Phys. 14, 1 (2017).

${ }^{16}$ J. W. M. Bush, "Pilot-wave hydrodynamics," Annu. Rev. Fluid Mech. 47, 269-292 (2015).

${ }^{17}$ J. W. M. Bush, A. U. Oza, and J. Moláček, "The wave-induced added mass of walking droplets," J. Fluid Mech. 755, R7 (2014).

${ }^{18}$ D. M. Harris and J. W. M. Bush, "Generating uniaxial vibration with an electrodynamic shaker and external air bearing," J. Sound Vib. 334, 255-269 (2015).

${ }^{19}$ J. Miles and D. Henderson, "Parametrically forced surface waves," Annu. Rev. Fluid Mech. 22(1), 143-165 (1990).

${ }^{20}$ D. M. Harris, T. Liu, and J. W. M. Bush, "A low-cost, precise piezoelectric droplet-on-demand generator,” Exp. Fluids 56(4), 83 (2015).

${ }^{21}$ J. Moláček and J. W. M. Bush, "Drops walking on a vibrating bath: Towards a hydrodynamic pilot-wave theory," J. Fluid Mech. 727, 612-647 (2013).

${ }^{22} \emptyset$. Wind-Willassen, J. Moláček, D. M. Harris, and J. W. M. Bush, "Exotic states of bouncing and walking droplets," Phys. Fluids 25(8), 082002 (2013).

${ }^{23}$ G. Pucci, D. M. Harris, L. M. Faria, and J. W. M. Bush, "Walking droplets interacting with single and double slits," J. Fluid Mech. 835, 1136-1156 (2018).

${ }^{24}$ P. A. Milewski, C. A. Galeano-Rios, A. Nachbin, and J. W. M. Bush, "Faraday pilot-wave dynamics: Modelling and computation," J. Fluid Mech. 778, 361-388 (2015)

${ }^{25}$ F. Moisy, M. Rabaud, and K. Salsac, "A synthetic schlieren method for the measurement of the topography of a liquid interface," Exp. Fluids 46(6), 1021 (2009).

${ }^{26}$ A. P. Damiano, P.-T. Brun, D. M. Harris, C. A. Galeano-Rios, and J. W. M. Bush, "Surface topography measurements of the bouncing droplet experiment," Exp. Fluids 57(10), 163 (2016).

${ }^{27}$ L. M. Faria, "A model for Faraday pilot waves over variable topography," J. Fluid Mech. 811, 51-66 (2017).

${ }^{28}$ J. Moláček and J. W. M. Bush, "Drops bouncing on a vibrating bath," J. Fluid Mech. 727, 582-611 (2013).

${ }^{29}$ G. Pucci, P. J. Sáenz, L. M. Faria, and J. W. M. Bush, "Non-specular reflection of walking droplets," J. Fluid Mech. 804, R3 (2016).

${ }^{30}$ A. Eddi, S. Eric, J. Moukhtar, E. Fort, M. Rossi, and Y. Couder, "Information stored in Faraday waves: The origin of a path memory," J. Fluid Mech. 674, 433-463 (2011).

${ }^{31}$ A. U. Oza, R. R. Rosales, and J. W. M. Bush, "A trajectory equation for walking droplets: Hydrodynamic pilot-wave theory," J. Fluid Mech. 737, 552-570 (2013).

${ }^{32} \mathrm{M}$. Labousse and S. Perrard, "Non-Hamiltonian features of a classical pilot-wave dynamics,” Phys. Rev. E 90(2), 022913 (2014). 